CAHIERS DE

NARRATOLOGIE

\section{Cahiers de Narratologie}

Analyse et théorie narratives

$36 \mid 2019$

Rhétorique et représentations de la culture mafieuse. Images, rituels, mythes et symboles

\title{
Les Beati Paoli : genèse d'un mythe
}

\section{Francesco Valerio Tumbarello}

Translator. Manuela Bertone (de l'italien)

\section{(2) OpenEdition Journals}

Electronic version

URL: http://journals.openedition.org/narratologie/9641

ISSN: 1765-307X

Publisher

LIRCES

Electronic reference

Francesco Valerio Tumbarello, «Les Beati Paoli : genèse d'un mythe », Cahiers de Narratologie [Online], 36 | 2019, Online since 20 December 2019, connection on 20 December 2019. URL : http:// journals.openedition.org/narratologie/9641

This text was automatically generated on 20 December 2019.

Article L.111-1 du Code de la propriété intellectuelle. 


\title{
Les Beati Paoli : genèse d'un mythe
}

\author{
Francesco Valerio Tumbarello
}

Translation : Manuela Bertone (de l'italien)

\section{Introduction}

1 On dit que dans la première moitié du XVIIIe siècle, une secte de justiciers, les Beati Paoli, a été fondée à Palerme pour défendre les plus faibles face aux abus des puissants. Dans le quartier du Capo, dans une grotte souterraine, se trouve leur tribunal. La mémoire populaire s'en souvient en ces termes :

le jour, pour mieux comprendre ce qui se passait, ils s'habillaient comme les moines de Saint François de Paule et se tenaient dans les églises pour (faire semblant de) prier le chapelet : la nuit, ils se réunissaient et discutaient de ce qu'ils avaient vu et appris, et ils décidaient des vengeances ${ }^{1}$.

Cette secte a été décrite par l'écrivain palermitain Luigi Natoli qui, en 1909-1910, sous le pseudonyme de William Galt, publie dans le «Giornale di Sicilia » un feuilleton en 239 épisodes intitulé I Beati Paoli ${ }^{2}$. Conçu lors des mobilisations pour la défense de la respectabilité de l'île, suite au procès pour le meurtre d'Emanuele Notarbartolo, l'ancien maire de Palerme et directeur du Banco di Sicilia, qui avait eu lieu en 1893, et la création du comité Pro-Sicilia (1902)3 ${ }^{3}$, dont Natoli fut l'un des promoteurs, ce roman est un véritable «exutoire du libéralisme sicilien $»^{4}$ contre l'opinion dominante en Italie selon laquelle la mafia est une coutume propre à la Sicile. Comme tous les romans populaires, I Beati Paoli est plutôt réconfortant que révolutionnaire, et part du principe que «s'il y a des contradictions sociales, il y a des forces qui peuvent y remédier $»^{5}$. L'auteur a souvent été accusé « d'avoir alimenté en termes apologétiques ce que Pitrè appelait le sentiment mafieux $»^{6}$, parce qu'il a donné «à la mafia une aura de légitimité ancrée dans l'imaginaire populaire $»^{7}$. En effet, grâce à Natoli, la mystérieuse société des Beati Paoli, «dont on parlait avec terreur et respect et dont les jugements étaient exécutés sans faille par une main que personne ne voyait $\|^{8}$, s'affirme comme "prototype de la mafia ", une société tout aussi secrète, fondée à son tour sur la terreur et le respect. La mise en scène scrupuleuse de la réalité sociale et topographique de la capitale sicilienne au XVIIIe siècle ainsi que le savant mélange de 
personnages réels et inventés, génèrent une superposition entre vérité et fiction qui, dans l'imaginaire collectif, acquiert une cohérence historique incontestable. Pour des générations de lecteurs, qui en font un succès durable, toutes classes sociales confondues, le roman est bel est bien un «document ». Autrefois, il était lu par le « pater familias [...] dans les longues soirées d'hiver [...] d'une voix voilée d'émotion [...] aux parents et aux voisins qui l'entouraient et l'écoutaient dans un silence religieux ${ }^{10}$. Aujourd'hui encore, les réimpressions sont vite épuisées. Jean-Noël Schifano, dans une critique de la traduction française, en parle comme du «cinquième monument historique de la littérature italienne contemporaine $»^{11}$. Les Beati Paoli, forts de leur consécration littéraire, sont ainsi devenus un emblème de l'histoire sicilienne.

\section{Les déclarations des Beati}

3 Le sociologue Diego Gambetta se souvient qu'on lui avait conseillé de lire I Beati Paoli et la suite, Coriolano della Floresta (1914) s'il voulait comprendre Palerme ${ }^{12}$. Les anthropologues américains Peter et Jane Schneider rapportent une expérience similaire : «Au milieu des années 60, dans une ville rurale de la Sicile intérieure, un mafieux local nous a recommandé de lire le livre I Beati Paoli si nous voulions vraiment saisir "l'esprit de la mafia" »". Dans les mêmes années, on s'arrachait les romans de Natoli même dans la prison palermitaine de l'Ucciardone ${ }^{14}$. Bien que semi-analphabète, le chef de Cosa Nostra Salvatore Riina connaissait par cœur les faits et gestes des Beati Paoli. En 1963, lorsqu'il partage sa cellule avec Gaspare Mutolo, il lui en recommande la lecture. Ce dernier, devenu collaborateur de justice, déclare devant la commission parlementaire anti-mafia avoir trouvé dans le roman I Beati Paoli les anciennes valeurs de la mafia de son adolescence, capable de sagesse et discernement, bien différente de la mafia impitoyable des Corléonais ${ }^{15}$. L'avocat américain de Gaetano Badalamenti, lors du procès "Pizza Connection ", utilise à son tour la légende des justiciers mythiques lorsqu'il prétend que son client n'a rien à voir avec la mafia de la drogue que dirige Riina, car il appartient à la mafia issue de «l'ancienne tradition Beati Paoli »" Tommaso Buscetta, le boss mafieux qui, en 1984, a révélé au juge Giovanni Falcone l'organigramme de la mafia, relate que lors de sa détention à l'Ucciardone, il y avait un détenu qui connaissait par cœur les deux romans de Natoli et qui en proposait une excellente mise en scène : « Le soir, quand les cellules étaient fermées, on l'emmenait à l'infirmerie, où les chefs de la mafia étaient installés dans le confort dû à leur rang, et il était censé les divertir avec son spectacle $»^{17}$. Quand il a fini de purger sa peine, les chefs mafieux se sont opposés à sa libération, exigeant qu'il reste pour les amuser. Sa libération a effectivement été reportée de quelques jours pour lui permettre de terminer l'histoire.

4 Le mafieux palermitain Salvatore Contorno, connu pour son talent et son courage, était surnommé "Coriolano della Floresta $»^{18}$. Le collaborateur de justice Salvatore Cucuzza se souvient des préceptes que l'on inculquait aux néophytes, au nom des Beati Paoli : « respecter les membres du clan, les femmes et les enfants, autrement dit protéger quiconque était en état de faiblesse $»^{19}$. Buscetta aussi se souvient de l'enseignement reçu: «Les anciens, les vieux mafieux qui m'ont éduqué et expliqué les traditions de Cosa Nostra quand j'avais moins de vingt ans, m'ont dit qu'elle était née pour défendre les faibles des abus des puissants $\aleph^{20}$. En traçant la lignée généalogique de l'organisation, il ajoute: «La mafia n'est pas née maintenant: elle vient du passé. 
D'abord il y a eu les Beati Paoli qui ont combattu avec les pauvres contre les riches, puis les Carbonari : nous avons le même serment, les mêmes devoirs " ${ }^{21}$. La mafia de Catane est également convaincue que les hommes d'honneur sont les descendants des Beati Paoli. D'après le collaborateur de justice Antonino Calderone, Cosa Nostra est « née au temps des Vêpres siciliennes : le peuple s'est rebellé et les Beati Paoli sont nés aussi. Les hommes d'honneur font référence aux Beati Paoli, à partir des émeutes de Palerme $»^{22}$. Leonardo Vitale, l'un des premiers «repentis» de Cosa Nostra, a décrit en 1973 le cérémonial de son affiliation à la famille Altarello de Baida : devant les autres affiliés, le zio lui avait piqué l'index avec une épine d'oranger amer et avait brûlé une image pieuse, lui faisant répéter « le rite sacré des Beati Paoli $»^{23}$. On peut enfin rappeler que lors du procès de Cosa Nostra, Salvatore Riina et Gaspare Mutolo, confrontés, s'accusent mutuellement en citant des faits et des personnages tirés de l'œuvre de Natoli. Riina menace implicitement Mutolo en l'accusant d'être un nouveau Matteo Lo Vecchio (à savoir le policier exécuté par les Beati Paoli puisque coupable de trahison), tandis que Mutolo reproche à Riina et à sa famille d'avoir trahi l'esprit authentique et traditionnel des Beati Paoli ${ }^{24}$.

Ces renvois répétés aux symboles, aux rites et aux objectifs des Beati Paoli pourraient constituer une preuve de la continuité historique qui lie cette secte légendaire et la mafia ; mais ils pourraient aussi prouver qu'afin de « renforcer et légitimer sa propre identité ${ }^{25}$, la mafia a sciemment construit sa propre tradition en exploitant le chevauchement qui s'est créé entre la représentation sociale de la mafia et la légende de la secte des justiciers. Cette invention de la tradition est une pratique partagée par les institutions et les groupes sociaux. Comme l'a souligné Eric Hobsbawm, elle remplit trois fonctions importantes: "celle d'établir ou symboliser la cohésion sociale ou l'appartenance à des groupes; celle de légitimer des institutions, des statuts ou des relations d'autorité; celle d'inculquer des croyances, des systèmes de valeur et des codes de conduite ${ }^{26}$. S'auto-identifiant dans la secte mythique des Beati Paoli, la mafia s'est dotée d'un passé glorieux, fondant sa genèse sur une filiation historique fictive.

\section{Les origines des Beati Paoli}

Depuis deux siècles, les érudits recherchent des preuves de l'existence des Beati Paoli, mais à ce jour personne n'a de certitudes. La seule source documentaire est constituée par quelques pages manuscrites du marquis de Villabianca, historien palermitain, datant de 1790, que l'on considère comme la pierre angulaire de toute reconstruction historique concernant la secte. En attendant que d'autres documents soient repérés, l'historien Francesco Renda a précisé qu'il est nécessaire de déterminer à quel moment et de quelle manière cette «imposante tradition orale et écrite $»^{27}$ a pu être construite. Renda lui-même a fait connaître tout le matériel qu'il a accumulé en étudiant l'évolution de la tradition de la légende des Beati Paoli et a pu identifier quatre étapes fondamentales: 1790, année de publication du troisième volume de la traduction (signée de Scasso Borrello) du livret du marquis de Villabianca et du journal de voyage de Friedrich Münter ; 1836 : année de publication de la nouvelle I Beati Paoli de Vincenzo Linares et des Lettere de l'officier Quattromani ; 1873-75: années au cours desquelles, d'une part, le conseil municipal de Palerme dédie aux Beati Paoli la rue et la place où l'on supposait qu'ils s'étaient réunis, et d'autre part, Bruno Arcaro, dans un essai paru dans l'hebdomadaire palermitain « Libertà e Diritto », retrace la légende des Beati Paoli 
sous un jour autonomiste, élevant ainsi les membres de la secte au rang de champions de la lutte politique, tandis que Giuseppe Pitrè publie les versions orales de la légende racontées par Francesca Campo ; 1909-1910, années de publication du feuilleton I Beati Paoli de Luigi Natoli.

7 Pionnier de la recherche sur les Beati Paoli, Francesco Paolo Castiglione en voit les premières traces dans les révoltes siciliennes de la première moitié $\mathrm{du} \mathrm{XVI}{ }^{\mathrm{e}}$ siècle et relie la secte à la confrérie impériale des Sept Anges, «la plus importante et la plus mystérieuse des confréries de la Sicile au XVIe siècle ${ }^{28}$, composée de marchands pisans installés en Sicile qui, avec l'appui des minimes de Saint François de Paule, allaient consolider leur pouvoir politique à Palerme au cours des siècles suivants. C'est précisément de cet ordre religieux que dériverait le nom de Beati Paoli, « en tant que déformation de l'expression Biat'i Paula, c'est-à-dire le Bienheureux de Paola », le nom utilisé pour désigner François de Paule «entre sa mort et sa canonisation ${ }^{29}$. L'association des Beati Paoli ne serait donc pas née dans le but de protéger le peuple mais, au contraire, avec l'objectif politique de veiller aux intérêts des classes dirigeantes. Fidèle à l'enseignement et aux recherches érudites de Salvatore Salomone Marino, Castiglione s'inspire du récit oral de Francesca Buscemi de Palerme (recueilli et publié en 1876 par Salomone Marino) : victime de la famine, le peuple rebelle parvint à reprendre possession du blé que juges et puissants avaient caché ; une fois la révolte terminée, les juges en colère prirent au piège une vingtaine de personnes et les massacrèrent; lorsqu'il apprit la nouvelle, le peuple en colère obligea les juges à fuir à Messine pour se mettre sous la protection du vice-roi, mais près d'une église deux Beati Paoli, déguisés en mendiants avec le chapelet à la main, tuèrent les juges et vengèrent ainsi le peuple.

8 Castiglione indique qu'à la base de cette histoire, que " pendant des siècles, le peuple avait transmise oralement $»^{30}$, il y a l'expulsion du vice-roi Moncada (1516) et la révolte de Squarcialupo (1517). D'après Castiglione, la formule « se ne diedero più che i Beati Paoli » [ils se rouèrent de coups pire que les Beati Paoli] contenue dans les Ragionamenti (1534-1536) de Pierre Arétin, est une preuve irréfutable de l'existence de la secte : cette expression aurait été inventée après l'épilogue tragique de la révolte menée par le gentilhomme Gian Luca Squarcialupo le 8 septembre 1517 dans l'église de l'Annunziata à Palerme, où un groupe d'individus «en robe de bure et capuche $»^{31}$, proches de l'oligarchie locale, avait éliminé ce même Squarcialupo et ses compagnons. Selon Salomone Marino aussi, la présence de la phrase chez l'Arétin montre « que la tradition des Beati Paoli était également très connue sur le continent, à tel point qu'elle était devenue proverbiale $»^{32}$ et qu'il faudrait donc la faire remonter à une époque antérieure à celle qu'avait retenue Villabianca. « Dare i beati paoli » [filer les beati paoli] voulait dire «infliger une correction solennelle, abominable, terrible, maltraiter horriblement, y compris au sens moral $»^{33}$.

9 La secte est brièvement mentionnée dans l'œuvre monumentale de Carmelo Trasselli, qui avance l'hypothèse que les Beati Paoli sont des descendants des Cirauli, les enchanteurs de serpents " aux pouvoirs mystérieux et occultes d'après le petit peuple, dont l'occultisme dégénère ensuite au XVIIIe siècle et les transforme en une une mystérieuse secte des vengeurs et d'assassins» ${ }^{34}$. Selon cet historien sicilien l'évocation des Beati Paoli chez l'Arétin « constituerait la trace la plus ancienne du nom d'une secte clandestine ayant évolué à Palerme au XVIIIe siècle $»^{35}$. 
10 L'historien vénitien Marino Berengo a signalé qu'à Venise l'expression «c' ne è anche per $i$ Beati Paoli » [il y en a même pour les Beati Paoli] est utilisée « en servant un plat pour en indiquer l'abondance et rassurer ainsi les convives, qui ne manqueront pas de nourriture ${ }^{36}$. À partir de la lecture du récit de Francesca Campo, Berengo estime qu'à l'origine de l'expression vénitienne il pourrait justement y avoir la fantomatique secte palermitaine.

11 La présence d'une expression similaire en langue parlée dialectale a été observée en Corse. Pendant l'occupation française, les disciples de Pasquale Paoli avaient formé une organisation patriotique et contre-maçonnique, et l'avaient appelée Beati Paoli, d'après l'association «formée à Palerme au milieu du XVIIIe siècle, qui regroupait des défenseurs zélés de la foi, inquiets des progrès du rationalisme $»^{37}$. Son nom ne dériverait donc pas du nom de famille du patriote corse, mais de la «société sicilienne des Vengeurs, car les liens entre les sociétés de ces deux îles sont l'une des clés de leurs histoires secrètes respectives $»^{38}$. En Corse, «si l'on en croit la mémoire populaire [la société est] apparue plus tard, lors de la révolte de la Crucetta $»^{39}$, à savoir en 1798. La mémoire populaire, vivace dans la langue parlée, se rappelle que «les Beati Paoli devaient être extrêmement nombreux puisque aujourd'hui encore, dans la Castagniccia, on dit pour parler d'une multitude de gens: "Sô quant'e i Beati Paoli !" [ils sont aussi nombreux que les Beati Paoli] $»^{40}$.

12 Mais relier l'expression «Beati Paoli » à la secte palermitaine du même nom signifie entamer une analyse ex post, à partir de la description des Beati Paoli fournie par le Marquis de Villabianca. En nous concentrant sur le caractère performatif et métaphorique du langage et sur sa capacité à structurer et façonner la réalité, nous souhaitons au contraire procéder ex ante, en examinant les significations de l'expression Beati Paoli au cours des siècles, afin de vérifier si la tradition a pu se former à partir d'une expression préexistante.

\section{Au-delà de la signification traditionnelle}

L'expression «Beati Paoli » n'est autre qu'un est un latinisme liturgique, l'un des nombreux latinismes présents dans les langues vernaculaires de l'Italie ${ }^{41}$. Elle est dérivée du génitif singulier Beatus Paulus que l'on répète lors de la lecture liturgique des Épîtres de Saint Paul: Lectio epistolae Beati Pauli Apostoli42. Ignorant la valeur grammaticale du génitif latin, les locuteurs ont interprété l'expression Beati Pauli comme une forme de pluralité et, par conséquent, l'ont associée à l'idée d'abondance. Comme beaucoup de latinismes liturgiques, cette locution a perdu sa valeur sacrée originelle pour acquérir de nouveaux contenus sémantiques, se transformant en expression idiomatique. Les expressions idiomatiques étant des «expressions polylexicales qui combinent un signifiant fixe avec une signification conventionnelle typiquement non littérale $»^{43}$. «Beati Paoli », en tant qu'expression idiomatique, a les caractéristiques suivantes: elle est figée, c'est-à-dire dénuée de flexibilité syntaxique sans pour autant perdre son sens conventionnel; elle n'est pas ambiguë car elle n'admet que de sens figuré ; elle n'est pas compositionnelle car le sens figuré ne peut être dérivé du sens propre des termes qui la composent ; elle est opaque car, à partir du sens littéral, elle ne permet pas de remonter facilement au sens idiomatique. Quant à sa motivation, c'est-à-dire l'histoire qui l'a générée ${ }^{44}$, elle n'est pas univoque, car, si on peut la considérer comme étant intertextuelle, puisque tirée de lectures liturgiques en 
latin, elle est aussi symbolique, puisque sa signification figurée pourrait dériver du symbolisme de Saint Paul en tant que dispensateur de dons. Une ancienne croyance populaire attribue au 25 janvier, jour de la conversion de Saint Paul, le pouvoir de prédire le résultat de la moisson de l'année qui commence, et l'appelle San Paolo dei segni [Saint Paul des signes $]^{45}$.

Si en Sicile l'expression « Beati Paoli » renvoie à la secte secrète, une analyse diatopique nous a permis de constater que dans de nombreuses régions italiennes il s'agit d'une locution indiquant l'abondance ou une grande quantité. Par ailleurs, l'analyse diachronique de nombreuses œuvres (à partir du XVIe siècle) appartenant à différents genres littéraires, pour la plupart en langue vernaculaire, nous a permis d'établir que la plus ancienne source de l'expression idiomatique «Beati Paoli» au sens d'« abondance » remonte effectivement à 1534 (ce sont les Ragionamenti de l'Aretin déjà évoqués ci-dessus). Le traité linguistique de Benedetto Varchi, L'Ercolano (1570), qui énumère plusieurs formes argotiques et idiomatiques, lui attribue la même signification : «chi favella sine fine dicentes, dice più cose che non sono $i$ beati Pauli $»^{46}$ [celui qui parle sans fin, dit tout plein de choses]. L'expression est présente dans le poème burlesque Il Malmantile Racquistato (1676) de Lorenzo Lippi, qui contient une longue série de proverbes et d'expressions populaires florentines : la reine Celidora, occupée à regagner son royaume, se retrouve "in un campo pien di cavoli / n'affettò tanti che Beati Pavoli $\aleph^{47}$ [dans un champ plein de choux où elle en trancha une énorme quantité]. À Naples tout comme à Florence, l'expression signifie " abondance ", comme en témoigne la première églogue de Le Muse napoletane (1635) où Giambattista Basile décrit deux frimeurs qui finissent par se disputer et se menacer mutuellement: "si te mecco sse mmano/a duosso, ne averai / pe le beate Paole $!_{\aleph^{48}}$ [si je te frappe, je t'en mettrai tout plein]. En Sicile aussi, l'expression idiomatique «Beati Paoli » signifie «abondance »: dans un recueil poétique de Girolamo D'Avila, Canzoni amorosi (première moitié du XVIe siècle), on remarque un commentaire ironique à la pénurie alimentaire dont souffrent les moines dans un couvent: "Su ricchi l'hurtulani; a li pignati / ndanzanu quanto li beati Pauli $»^{49}$ [les légumes/les moines sont riches et dans les cocottes ils dansent en abondance]. Tout au long du XVIIe siècle aussi, l'expression Beati Paoli apparaît dans des contextes narratifs qui font allusion aux coups. Le peintre et poète syracusain Girolamo Comes (1527-1591), dans Lu bravazzu, le poème où il raconte les exploits du protagoniste aux prises avec quarante soldats, écrit : «Nd'happiru quantu li beati pauli » ${ }^{50}$ [il ont pris une sacrée raclée]. Dans Gli amorosi inganni (Paris, 1609) de Vincenzo Belando, Catonzo, le protagoniste, raconte ses aventures de jeunesse, notamment une expérience professionnelle en tant que confiseur qui a tourné court car il mangeait les dragées au lieu d'en produire: "lu mastru chi sind'adunau, e pigliatu un bastuni mindi dunau quanti li beati pauli $\aleph^{51}$ [le chef s'en aperçut, il prit un bâton et m'asséna un tas de coups]. Le poète Giuseppe Scimeca (disparu en 1645), auteur de sonnets recueillis et publiés à titre posthume par Giuseppe Galeano, l'utilise dans cette locution : "Amicu miu si troppu mi dunniju / Iu nd'havirò di li beati Pauli, / Li griddi in testa, à quantu cosi viju / Mi vannu sbardi sbardi comu ciauli $\aleph^{52}$ [mon ami, si j'écris trop, j'aurai des ennuis en quantité, dans ma tête je vois tant de choses, et des grillons qui cancanent comme des corbeaux]. Enfin, dans La Cuccagna conquistata (1640) du Palermitain Giuseppe Della Montagna, Nino, le protagoniste, tue Cola Cutiddazzu qui harcèle sa femme Betta, parvient à s'échapper et, avec l'aide de Zoroastro, part pour l'île d'abondance et s'exclame: "Iamu in Cuccagna; ma si semu visti, / $N d$ 'havemu quantu li biati pauli " ${ }^{53}$ [Allons au pays de cocagne; mais si on nous voit, on nous colle une grosse castagne]. 
Essentiellement, tout au long du XVIIe siècle, l'expression idiomatique «Beati Paoli » apparaît dans des contextes narratifs où sa signification (et par conséquent son interprétation) n'est autre que "abondance de coups». Le lien conceptuel entre l'expression «Beati Paoli» et les "coups» qu'elle sous-entend a donc imposé la spécification "abondance de coups». Cela est confirmé par le Dizionario siciliano e italiano (deuxième moitié du XVIIe siècle) dont l'auteur est le philologue palermitain Placido Spatafora, où l'entrée «Pauli » renvoyant au dicton « cinni detti di li biati Pauli, o quantu li biati Pauli » est expliquée comme suit: "dari una sugghiata di vastunati " $^{54}$ [donner une bonne raclée]. Cette spécification est encore plus évidente dans des œuvres siciliennes de la première moitié du XVIIIe siècle, comme par exemple dans les vers de Vincenzo Gagliano "E sempri dissi sintennu li ciauli, / Già mi darrannu li biati Pauli » ${ }^{55}$ [et je dis toujours en entendant les corbeaux, qu'il me donneront un tas de coups].

Une signification similaire se dégage des vers de Biagio Minaci (1744) : «Appuntu fannu comu li Cirauli / Ch'a tutti ciarmanu cu lu so chiamu / Pri darici poi biati pauli » ${ }^{56}$ [Justement, ils font comme les Cirauli, qui attirent les gens avec leur chant, et ensuite leur donnent un tas de coups]. Dans les deux cas, la locution «Biati Pauli » se réfère à l'abondance de coups, mais, à la différence de ce qui se produit dans les textes du XVIIe siècle, elle n'est pas introduite par «di» ou «quantu»: elle n'est plus complément indirect mais complément d'objet direct, et peut être utilisée en guise de synonyme de "coups abondants ". Cette transformation, due à une resemantisation contextuelle ${ }^{57}$, a entraîné un rétrécissement sémantique ${ }^{58}$ de la locution ainsi qu'une ré-motivation de type métonymique. Comme chacun sait, la métonymie n'est autre qu'un processus de substitution à l'intérieur d'un même domaine, où une expression est utilisée pour désigner une deuxième entité à laquelle elle est étroitement lié ou contiguë. En l'espèce, l'élément « Beati Paoli » est utilisé pour indiquer aussi la deuxième entité, les " coups ». Par conséquent, le binôme "Beati Paoli », désormais autonome, est entifié. Il ne représente plus une entité abstraite telle que l'abondance, mais une entité concrète : les coups en grande quantité.

\section{Les voyageurs du Grand Tour}

17 À partir de la seconde moitié du XVIIIe siècle, la Sicile, jusqu'alors considérée comme une terre lointaine et dangereuse ${ }^{59}$, est incluse dans l'itinéraire du Grand Tour en vogue parmi les élites européennes, et elle devient étape obligée de la redécouverte du monde grec. Dans un court essai de 1759 consacré aux temples d'Agrigente, Johann Joachim Winckelmann ${ }^{60}$ attire l'attention sur l'architecture dorique de l'île. Le voyage en Sicile, qu'il avait planifié à plusieurs reprises sans jamais le réaliser, fut effectué en 1767 par son élève Johann Hermann von Riedesel, auteur d'un journal à succès publié en allemand en 1771 et immédiatement traduit en français et en anglais. Ouvrage de référence de la littérature odeporique, ce texte contient tous le topoi destinés à façonner l'idée de la Sicile dans l'imaginaire culturel européen entre le XVIIIe et le XIXe siècle. Von Riedesel s'attarde également sur les coutumes, les traditions et le caractère des autochtones. C'est sans doute à Trapani qu'il entend parler des péripéties d'une mystérieuse confrérie de Saint Paul, née au XVIIe siècle, du temps de l'empereur Charles Quint, qu'il présente en ces termes :

Voici un exemple qui vous prouvera jusqu'à quel point les Siciliens sont portés à la vengeance, \& les traces profondes qu'a laissées chez eux l'ancien esprit républicain. 
Du temps de l'empereur Charles $V$, il se forma à Trapani une confrairie sous le nom de confraternita di San Paolo, dont l'institution \& le vœu consistoient à prononcer des jugemens sur les actions \& la conduite de leurs magistrats, de leurs concitoyens \& de chaque habitant de la ville ; quiconque avoit été condamné par toute l'assemblée étoit perdu sans ressource, \& celui des membres de la confrairie que l'on chargeoit de l'exécrable fonction d'assasin, étoit obligé d'obéir sans replique, \& d'expédier en cachette cet homme ainsi condamné secrettement par cet abominable tribunal ${ }^{61}$.

18 À l'origine de ce récit, dont il n'existe aucune trace dans l'histoire de Trapani ou de la Sicile, il y a probablement l'une des nombreuses histoires siciliennes de brigands ou de révolte populaire qui circulaient à l'époque et que les gardes racontaient aux voyageurs illustres qu'ils accompagnaient durant leurs déplacements. Comme le rappelle Patrick Brydone, les gardes voulaient les impressionner en leur parlant du chemin parcouru: « ils nous montroient à chaque mille l'endroit où un homme avoit été volé, \& plus loin, le chemin où un autre avoit été assassiné [...] pour se donner un air d'importance \& obtenir de nous une plus grande somme d'argent $"^{62}$. ÀTrapani, à cette époque, on se souvient encore des prouesses du bandit originaire de Mazara del Vallo Antonio Catinella (1675-1706) surnommé Sata li viti [saute les vignes] pour son agilité: «il recherchait les gens riches et [...] il faisait beaucoup de bien aux pauvres; sans jamais les agresser, il les secourait $"^{63}$. Après un siècle environ, à Mazara del Vallo, la tradition locale en parle encore comme d'un homme bon et généreux qui " protégeait les veuves et les enfants; vengeait les torts infligés aux faibles par les puissants » en exerçant " cette justice privée qui, parfois, est justifiée et rendue quasiment nécessaire par la faiblesse des lois $»^{64}$. Les deux histoires expriment une même idée de justice, tout en présentant des personnages et des époques différentes. Il est possible que von Riedesel se soit inspiré des révoltes qui ont caractérisé l'histoire de la Sicile sous Charles Quint, racontées par l'historien Thomas Fazello, ce qui expliquerait à la fois l'importance accordée au caractère républicain des Siciliens et le choix de faire évoluer la compagnie de Saint Paul sous l'empereur espagnol. Étant donné qu'en Sicile les actes de violence pouvaient être indiqués par l'expression «Beati Pauli ", on peut supposer que, parmi les nombreuses histoires entendues par von Riedesel, plusieurs aient repris cette expression qui, par son caractère atypique, a pu attirer son attention. Comme beaucoup de voyageurs de son temps, von Riedesel était francophone. Il est très probable que son accompagnateur-informateur, traduisant «Beati Pauli» ait utilisé l'expression raccourcie «Saints Pauls» au lieu de la traduction littérale, «Bienheureux Pauls». Von Riedesel a bien évidemment entendu l'expression «Saints Pauls» au singulier (Saint Paul) et c'est ainsi qu'il l'a interprétée, en imaginant un groupe de personnes réunies dans une fraternité paulinienne, comme il l'écrit dans la première édition allemande de son journal : «Brüderschaft vereiniget, welche sich di St. Paolo nannte » ${ }^{65}$. Quelle que soit l'histoire qui a influencé von Riedesel, il a bien saisi la nuance violente inhérente à l'expression «Beati Pauli » et a introduit une généralisation, en mettant les actions violentes sur le compte de la nature vindicative des Siciliens, de leur esprit républicain, comme c'est du reste le cas dans toutes les représentations de la Sicile de la même époque. Après avoir éliminé les personnages secondaires, von Riedesel décontextualise l'histoire et, dans les pages de son journal, il utilise le nom de confrérie de Saint Paul. Ainsi, pour la première fois, se matérialise l'image d'une association, d'une confrérie : l'expression "Beati Paoli», atypique et obscure, se met de côté et cède la place à sa propre représentation sociale. À ce jour, von Riedesel est la première source historique où les Beati Paoli, la Confrérie de Saint Paul, sont décrits comme une secte. 
19 Produites par deux processus cognitifs, l'ancrage et l'objectivation, les représentations sociales répondent au besoin humain fondamental de faire face à la nouveauté et de rendre familier ce qui paraît inconnu, anormal et étranger ${ }^{66}$. En rendant habituel ce qui est inhabituel, le premier processus transfère le nouvel élément dans les catégories cognitives du sujet, où il peut être comparé et interprété, tandis que le second processus le reproduit parmi les choses familières, où il peut être domestiqué et ensuite géré et contrôlé. C'est ainsi que von Riedesel, en utilisant le terme allemand « Brüderschaft » transforme des actions violentes, conventionnellement appelées "Beati Pauli », en une communion d'intention. Ensuite, liant cette confrérie à la nature vindicative et à l'ancien esprit républicain des Siciliens, il en objective les pratiques dans le noyau figuratif d'une cour de justice qu'il définit « abominable ». Ontologisant la locution, von Riedesel rompt avec le lexique sicilien, pour proposer un nouveau schéma conceptuel dans le cadre duquel il la reconfigure et la déchiffre. Le signe linguistique devient ainsi figure et symbole d'une congrégation à laquelle il associe la métaphore du jugement et de la condamnation, qu'il naturalise et classe sous la rubrique institutionnelle du tribunal.

Il sera également traité de la sorte par d'autres voyageurs étrangers qui, dans leurs journaux, conservent inaltéré le noyau central de la représentation, même s'ils en donnent des interprétations différentes. Songeons, par exemple, au journal de l'économiste français Roland de La Platière, qui s'est rendu en Sicile en 1776 et qui mentionne l'histoire de la confrérie de Saint Paul de von Riedesel. Il critique l'auteur allemand pour ses réflexions sur la nature des Siciliens en s'efforçant de justifier l'action de la confrérie dans le cadre d'un contexte socio-économique qu'il essaie de restituer. À une époque où « la noblesse possède tout; elle gouverne presque; elle a le peuple à ses gages ${ }^{67}$, la condamnation à mort d'un individu qu'une assemblée a jugé indigne de faire partie de la société est le seul moyen dont disposent ceux qui n'ont aucun pouvoir, même si «ne falloit-il pas confondre l'amour de la liberté avec l'abominable rage de détruire sourdement son semblable ${ }^{68}$.

21 L'histoire de von Riedesel suscite beaucoup d'intérêt en Europe, surtout en France, à tel point qu'elle est publiée en annexe à la traduction française du journal de voyage de Patrick Brydone en Sicile, sous la direction du comte Jean-Nicolas Démeunier (1775) ${ }^{69}$, une édition largement diffusée et encore plus influente que la version anglaise, publiée à Londres 1773. Bien que dans ses lettres de Sicile Brydone ne mentionne pas l'histoire de von Riedesel, l'anecdote circule grâce à la traduction française du journal et devient célèbre à tel point qu'on la retrouve dans l'œuvre monumentale de l'abbé Joseph de La Porte, qui rassemble les descriptions les plus significatives de monuments, coutumes et anecdotes de pays lointains, tirées des meilleurs journaux de voyage de l'époque ${ }^{70}$. Son écho se répand également en Angleterre et en Allemagne, grâce aux journaux intimes des nombreux voyageurs qui se rendent en Sicile. Le théologien danois Friedrich Münter, qui, à l'automne 1785, y effectue un voyage politique et culturel, décrit dans son journal la «Confrérie de Saint Paul » et donne quelques détails nouveaux, précisant que le but de cette association consiste à "juger la conduite des autorités et des citoyens, mais surtout à protéger et venger les veuves, les orphelins et autres opprimés ${ }^{71}$. Il estime qu'il s'agit d'un vestige nuisible du passé, le résultat du despotisme espagnol, probablement répandu dans la Sicile tout entière, car « à Palerme on pouvait encore voir sous une haute voûte souterraine, l'endroit où avaient lieu ses réunions ${ }^{72}$. S'agissant des Beati Paoli en tant que tribunal, il évoque la Sainte-Vehme, 
la société secrète médiévale allemande, tristement célèbre pour ses jugements, remise en vogue par Goethe avec le drame Götz von Berlichingen (1773) et devenue l'un des piliers de l'imaginaire culturel et de la littérature gothique de la fin du XVIII ${ }^{\mathrm{e}}$ siècle $^{73}$.

La comparaison entre les Beati Paoli et la Sainte-Vehme est proposée par d'autres voyageurs, tels le poète allemand Stolberg, qui visite la Sicile en $1792^{74}$ ou le chevalier de Mayer, qui, suite à son voyage de 1791, décrit la confrérie de Saint Paul comme un prolongement de la Sainte-Vehme, une véritable «inquisition secrète » légitimée par l'autorité impériale :

Cette association était déjà en vigueur dans l'Allemagne: avait pour but d'arretter les progrès des Sectes, et l'esprit de révolte: on frapait en secret les victimes signalées ; parcequ'on n'avait pas assés d'autorité pour punir juridiquement : on ne voulait même pas laisser soupçonner qu'il éxistait des germes de rebellion dans les esprits $^{75}$.

Bouleversé par la Révolution française, de Mayer n'oublie pas d'ajouter des remarques réactionnaires contre les différentes formes de rébellion qui, surtout après 1789 , se propagent progressivement: "Tels sont le résultats des sectes, de l'anarchie, du fanatisme d'opinion, et du fanatisme de l'indépendance : le crime qui les produit est étouffé par des crimes $»^{76}$.

La représentation sociale forgée par von Riedesel et véhiculée par des voyageurs étrangers allait fournir aux intellectuels palermitains un schéma figuratif leur permettant de reconnaître dans l'expression «Beati Paoli » la trace linguistique d'une réalité préexistante dont il fallait rechercher les origines. Par ailleurs, la présence de la marque du masculin pluriel (Beati Paoli) portait à penser à une pluralité d'individus, en l'espèce des individus incarnant la violence. Le caractère performatif de la représentation, la subjectivité plurielle présente dans le sens littéral de la locution, ainsi que le sens figuré se rapportant à l'action violente, porteraient finalement à croire qu'une association de malfaiteurs était à l'origine de l'expression elle-même.

\section{La construction de l'imaginaire palermitain}

Tandis que les voyageurs étrangers consacrent plusieurs pages à l'ancienne Confrérie de Saint Paul, les érudits siciliens s'adonnent à la revalorisation du Moyen Âge et font réapparaitre la mystérieuse secte normande des Vendicosi. Giovanni Battista Caruso en donne une première description dans son Memorie istoriche di quanto è accaduto in Sicilia (1737), où il examine deux codex médiévaux, Chronicon Fossae Novae et Breve chronicon, précédemment publiés dans son Bibliotheca historica regni Siciliae ${ }^{77}$. Influencé par les études de théologie et d'histoire sacrée, il en fait une secte d'hérétiques : «À la fin de l'année 1186 [...] une secte mortifère de plusieurs hérétiques fut découverte parmi les vassaux du roi Guillaume, qui avait le nom de Vendicosi ou Vendicativi, dont le chef était un certain Adinulfo de Pontecorvo [...] à la faveur des ténèbres, ils se réunissaient pendant la nuit pour satisfaire de manière étrange tous leurs désirs $»^{78}$. Née dans «la Province au-delà du Phare ", c'est-à-dire au delà du détroit de Méssine, la secte "put facilement se répandre en Sicile $»^{79}$. Il convient de noter que dans les deux textes médiévaux, écrits par des auteurs originaires de Ceccano et de Cassino, il est question de faits, lieux et personnages de l'actuelle province de Frosinone, appartenant alors au Royaume de Sicile, mais aucune référence n'est faite à une éventuelle propagation de la secte en Sicile. Cependant, la corrélation entre les Vendicosi et la Sicile sera partagée 
par tous les écrivains siciliens. En 1769, elle réapparaît chez l'archevêque de Monreale Francesco Testa, qui mentionne comme lieu d'origine Latium de la secte ainsi que sa propagation ultérieure en Sicile ${ }^{80}$. Évoluant dans un climat culturel où l'hérésie n'est plus un souci obsédant, il détache les Vendicosi du cadre religieux propre à Caruso et les présente dans une perspective laïque: "Au Royaume de Sicile, on découvre une secte de fauteurs de trouble, appelée des Vindicativi ${ }^{81}$. Pour en expliquer le nom, il ajoute un nouvel élément: "ainsi nommés, car sous prétexte de venger les offenses d'autrui, ils commettaient toutes sortes d'iniquités $»^{82}$.

La version de Testa, dont l'autorité est alors indiscutable, sera acceptée et partagée par les intellectuels palermitains, parmi lesquels Mariano Scasso Borrello, un érudit qui a travaillé pendant des années à la traduction italienne de l'Histoire générale de Sicile de Jean Levesque de Burigny (1745). Dans le troisième volume (1790), lorsqu'il commente les codex médiévaux que Burigny s'était contenté de citer, il précise entre parenthèses et en italiques que les Vendicosi légitimaient leurs crimes «sous prétexte de réparer les torts des autres ${ }^{83}$. Il ajoute également en bas de page une note dans laquelle il met en corrélation la vengeance médiévale avec la célèbre expression "Beati Paoli", désormais "personnifiée » par la représentation sociale des voyageurs étrangers et connue dans tous les salons intellectuels de Palerme : «Il est notoire auprès du Peuple que cette Société secrète de vengeurs cachés, communément appelés les Beati Paoli, a été renouvelée à plusieurs reprises en Sicile et ailleurs ${ }^{84}$. Scasso Borrello attribue ainsi aux « vengeurs » de Palerme des images inhérentes à la représentation littéraire des «Vendicosi » du Moyen Âge: «ils allèrent jusqu'à les féliciter pour leur œuvre scélérate, comme si la tâche arbitraire de tuer qui bon leur semble, sous prétexte de venger les offenses subies par autrui, et de s'adonner avec davantage de force à l'administration de la justice, pouvait servir de justification à un délinquant, dans une Société bien réglée $»^{85}$. La traduction libre et les commentaires personnels de Scasso Borrello ouvrent la voie à la représentation sociale des Beati Paoli proposée par Villabianca. Et, contrairement à ce qu'affirme Renda, ils ne confirment pas l'existence "en Sicile d'une légende populaire orale concernant les Beati Paoli ${ }^{86}$. Bien au contraire, ils confirment l'influence exercée par von Riedesel sur les intellectuels de Palerme, puisque ces derniers associent l'expression «Beati Paoli» à des entités violentes très spécifiques, précisément après la publication de son journal.

Avec Wittgenstein, on pourrait dire que «une image les tenait captifs. Et ils ne pouvaient lui échapper, car elle se trouvait dans leur langage qui semblait la leur répéter inexorablement $»^{87}$. L'image qui les tenait captifs était celle qui donnait une cohérence ontologique au Beati Paoli : ils n'étaient plus une expression figurative, mais un sujet pluriel (suite à la représentation de la confrérie de Saint Paul), une image spéculaire de celle des Vendicosi esquissée par Testa.

Désormais, personne n'allait se demander ce que Beati Paoli voulait dire, mais qui ils étaient ou avaient été dans le passé. Il fallait donc leur donner un visage, les matérialiser, les fixer une fois pour toutes dans un lieu et un temps précis, mais toujours loin, à distance, comme pour en éloigner la cruauté.

C'est Francesco Maria Emanuele Gaetani, marquis de Villabianca (1720-1802), qui mettra noir sur blanc la base de la future légende des Beati Paoli, en mélangeant et harmonisant les éléments de différentes sources. Dans ses célèbres Diari (commencés en 1743, relatant plus de cinquante ans d'histoire palermitaine) il ne mentionne jamais les Beati Paoli. Ce n'est qu'en 1790, après la traduction italienne de l'œuvre de Burigny par 
Scasso Borrello, qu'il publie un court essai intitulé I Beati Paoli che successero agli antichi $v_{\text {vendicosi }}{ }^{88}$. La corrélation entre Beati Paoli et Vendicosi étant acquise, il évoque d'emblée la note de Scasso Borrello qui lui rappelle des souvenirs d'enfance : « Ces Beati Paoli sont des malfaiteurs, il me semble, d'après la tradition dont, enfant, j'ai entendu parler; il ne sont pas très anciens et ils ont fait parler d'eux dans la ville de Palerme sans doute plus qu'ailleurs " ${ }^{89}$. Les traces mémorielles de l'ancienne expression «Beati Paoli » sont désormais incarnées dans les justiciers-vengeurs d'une secte, et acquièrent en outre une matérialité historique, car ils correspondent à Giuseppe Amatore, pendu le 17 décembre 1704, et Girolamo Ammirata, pendu le 27 avril 1723. Sans oublier «le célèbre cocher de Palerme surnommé Vituzzu $»^{90}$, connu pendant l'enfance, « le dernier des délinquants de la Compagnie [...] des Beati Paoli $»^{91}$, rescapé au gibet parce qu'il s'était repenti et réfugié dans l'église de San Matteo. Lorsqu'il présente les objectifs de la secte, il rappelle que les puissants, notamment les Baroni (les grands propriétaires terriens), avaient l'habitude, à l'époque, de s'entourer de sbires et de faire la loi, tandis que les "gens modestes [...] dans l'impossibilité de rémunérer des hommes de main, $s^{\prime}$ enorgueillissaient de se défendre tout seuls, de leurs propres mains $»^{92}$. Dans le sillage de Caruso, qui, faisant le lien entre les Vendicosi et les Cathares, les appelait des hérétiques libidineux, Villabianca fait remonter les origines intellectuelles et religieuses des Beati Paoli aux Fraticelli franciscains qui, accusés d'hérésie, se réfugièrent en Sicile au XIVe siècle. De ces illustres prédécesseurs, le Beati Paoli auraient hérité les vertus pieuses et les mœurs chastes. Se questionnant sur l'étymologie du nom «Beati Paoli », il tente de remonter à l'origine de la secte. N'ayant malheureusement pas trouvé de sources historiques fiables, il suppose que le nom vient de Paul, au singulier, le prénom ou le surnom de l'ancien chef. Dans ce second cas, la référence aurait été précisément Saint Paul « qui, avant de devenir un vase d'élection, était un soldat $»^{93}$. Fier d'être comme Saint Paul, le chef " décida de l'imiter en faisant preuve de sainteté aussi, mais il voulut être les deux, à moitié : la journée, il était un homme pieux, allant à l'église, le chapelet à la main, ce pour quoi on l'appela Beato Paolo; la nuit, il était le chef des sbires, comme l'avait été Saint Paul lorsqu'il persécutait les Chrétiens $»^{94}$. Villabianca ne s'arrête pas là : après avoir donné une véritable consistance à la "congrégation infernale", il présente l'endroit où elle se réunit avec force détails : de la maison du juriste Giovan Battista Baldi «qui se trouve en Saint Côme dans la ruelle de Santa Maruzza, quartier du Capo ", l'on accédait à une grotte souterraine où autrefois "les membres de cette secte se rassemblaient et tenaient leurs réunions $[. .$.$] après minuit [. .$.$] pour que tout soit fait à la lumière de la$ bougie $»^{95}$.

La description des lieux sombres et des activités criminelles de la secte, semblables à celles du tribunal secret de la Sainte-Vehme, et de son objectif (la vengeance), qui est au cœur des descriptions des confréries de Saint Paul et des Vendicosi, font des pages de Villabianca un point de rencontre qui fera fonction de point de départ dans la représentation sociale des Beati Paoli à Palerme. Née dans le milieu intellectuel palermitain du XVIIIe siècle, cette représentation a immédiatement animé les conversations dans les salons, comme le confirme une page du journal de Léon Dufourny, architecte français vivant alors à Palerme, datée du 31 janvier 1791: «déjeuner chez Lioy, avec l'abbé Di Giovanni et Don Felice Visconti, architecte du duc de Monteleone. Nous avons parlé [...] de la Société des Beati Paoli, etc... ${ }^{96}$. C'est bien cette représentation sociale qui, rapidement, allait remplacer pour toujours, chez les 
locuteurs palermitains, la simple signification idiomatique d'une locution locale, jusqu'à devenir un mythe.

\section{Conclusion}

31 Après Villabianca, dont l'autorité scientifique sanctionne l'existence des Beati Paoli, d'autres auteurs commencent à écrire sur la secte, la considérant unanimement comme une réalité historique. Ainsi, par exemple, si en 1776 le prêtre palermitain Gaetano Alessi, dans sa collection manuscrite d'anecdotes siciliennes, s'était borné à reprendre l'histoire des Vendicosi telle qu'elle avait été racontée par l'archevêque Testa ${ }^{97}$, dans un recueil de 1803 il la développe en mettant en corrélation les Vendicosi et les Beati Paoli de Palerme, ce prouve l'influence du texte de Villabianca : «Vers la fin du XVIIe siècle et au début du XVIIIe siècle, c'est-à-dire depuis 1700, il était à Palerme une secte d'hommes dangereux, assassins, tueurs à gages, que le peuple nommait les Biati Pauli » ${ }^{98}$. Alessi identifie également le lieu de leurs réunions « dans une grotte souterraine située dans une maison en face du couvent de Saint Côme, appelée la Cuncuma »" D'après un manuscrit anonyme du XVIIe siècle, dans le quartier du Capo, en descendant de l'église de Saint Côme vers celle de Saint Jean à la Guilla, « il y avait un jardin appelé la Cuncuma, où il y avait une grande auberge où se donnait rendez-vous la pègre de Palerme, d'où l'expression "il est de la Cuncuma" pour désigner un homme sournois " ${ }^{100}$. C'est peut-être cela qui a conduit Villabianca à situer le tribunal secret au Capo. Quel meilleur lieu de réunion qu'un ancien puits thermique ${ }^{101}$ dans le jardin de la Cuncuma? C'est ainsi qu'il devient, dans l'imaginaire collectif, la grotte souterraine où se déroulent les réunions des juges assassins, avec ses grillages, ses entrées secrètes, ses autels et ses tables où l'on rend de terribles jugements. Et d'ailleurs, un siècle plus tard, le mot «cùncuma», en dialecte palermitain, désignera le lieu "de réunion occulte, où l'on décide crimes et séditions", tandis que le mot "cuncùmiu» indiquera un rassemblement d'individus, surtout en vue de « unions secrètes et mystérieuses $»^{102}$. Les environs aussi deviendront célèbres et seront utilisés comme des preuves incontestables de l'existence de la secte. De nos jours encore, les guides aidant, les touristes en raffolent.

Dans son guide de la ville publié en 1816, Gaspare Palermo, décrivait l'histoire de l'église Santa Maria del Gesù et soulignait la popularité de la ruelle adjacente, dite ruelle des Beati Pauli, où selon la tradition, «une association de tueurs et de malfaiteurs appelés Beati Pauli avait un réduit où tenir ses réunions, où on décidait à qui donner la mort $»^{103}$. Cette ruelle, que Villabianca appelle de Santa Maruzza, puisqu'elle se trouve à côté de l'église du même nom, s'appelait ruelle des Orphelins depuis que le Sénat de Palerme, au début du XVIIe siècle, avait octroyé l'église aux orphelins de Saint Roch, qui s'étaient installés dans les maisons adjacentes ${ }^{104}$. Manifestement, le récit de Villabianca a dû frapper les esprits des habitants du quartier du Capo, puisqu'ils en sont venus à re-nommer la ruelle de Santa Maruzza. Autrement dit, son récit a quitté les salons pour aller vers les quartiers populaires et s'installer dans la mémoire des lieux. Parallèlement, la représentation des Beati Paoli se propage et occupe une place de choix dans les pages des écrivains siciliens. En 1836, Vincenzo Linares publie une nouvelle consacrée au Beati Paoli dans le $n^{\circ} 35$ du journal Il Vapore et, dans le numéro suivant, un résumé des mémoires de Villabianca, en précisant que si la nouvelle était une fiction, «ceci est de l'histoire ${ }^{105}$. Et il termine son article en 
invitant les lecteurs à visiter les lieux de réunion de la secte, puisque la tradition était bien préservée par les habitants du quartier, à tel point que "chacun pourra vous indiquer, avec frayeur, ces endroits secrets $\aleph^{106}$. En 1840, Linares reprendra l'histoire des Beati Paoli pour l'augmenter et l'inclure dans ses Racconti popolari, en ajoutant une transcription partielle et infidèle du livret de Villabianca ${ }^{107}$. Peu importe: la représentation de la secte est ainsi étoffée d'éléments nouveaux qui vont enrichir la tradition orale palermitaine : la journée, les Beati Paoli se promenaient en robe de bure, comme des moines, pour recueillir les rumeurs, les confidences, les secrets de la ville; sur le coup de minuit, enveloppés d'un manteau noir, ils se rassemblaient dans la grotte de la Cuncuma pour désigner les coupables, planifier les vengeances, prononcer les condamnation à mort et gérér ainsi la justice à Palerme. Lors des réunions de ce tribunal secret « le magistrat corrompu, le noble arrogant, l'employé vénal, étaient mis en examen et jugés $»^{108}$. Les plaintes et les dénonciations étaient examinées, les injustices et les abus étaient sanctionnés : "Leurs jugements étaient brefs, pour la plupart approuvés par l'opinion, les votes étaient libres, les peines rapides et féroces. La voix du peuple est la voix de Dieux, disaient-il, et, tout comme Dieu, ils ne rendaient aucun compte à personne $»^{109}$. Le peuple avait trouvé en eux une autorité fiable, à l'écoute, capable de le défendre et d'appliquer la loi là où elle n'avait pas été respectée.

Le regard porté sur les Beati Paoli, encore résolument critique chez Linares, changera progressivement, au fil du temps, jusqu'à devenir bienveillant lors des luttes politique féroces qui déchirent la Sicile pendant les années qui suivent l'Unification italienne. Dans un territoire hâtivement perçu et classé comme mafieux, le mythe des Paoli Beati se lève pour défendre la dignité du peuple sicilien, pour devenir l'emblème de la lutte contre l'oppresseur et l'étranger.

Les liens entre la mafia, placée au centre du débat politique, et le mythe, investi de fortes connotations politiques, n'échappe pas à la Commission d'enquête parlementaire sur les conditions de la Sicile, qui, en 1875, voit précisément dans l'ancien tribunal des Beati Paoli les origines de la mafia :

Peut-être n'aurait-on pas tort de chercher les premières traces de la mafia dans cette secte des Vendicatori ou Beati Paoli, dont on se souvient jusqu'au début du XVIIIe siècle ; une secte obscure, qui nuitamment, comme la Sainte Wehme, jugeait à Palerme les nobles insolents et les magistrats corrompus et qui sans doute, ellemême corrompue, transforma en offensive organisée cette forme défensive que les populations utilisaient autrefois contre la violence de ceux qui les gouvernaient ${ }^{110}$.

Il n'est donc pas surprenant que les mafieux, désormais associés aux anciens vengeurs du peuple, aient décidé de s'auto-identifier comme les descendants de ces juges féroces et de prendre possession de leur légendaire siège historique : environ un mois avant la publication par Natoli du premier épisode du feuilleton I Beati Paoli (6 mai 1909), un rapport de police daté du 11 avril 1909, indiquait que la puissante organisation criminelle de la Mano Nera [main noire] organisait habituellement ses réunions dans une maison adjacente à l'église de Santa Maruzza, au « coin de la ruelle des Orphelins » 111 . 


\section{BIBLIOGRAPHY}

Alessi, Gaetano (1776), Notizie piacevoli e curiose o aneddoti dilettevoli, ed eruditi intorno a diversi riti, costumanze, fatti, epoche, di alcune cose rimarchevoli appartenenti a Palermo ed alla Sicilia, Biblioteca Comunale di Palermo, ms. Qq H 43.

Alessi, Gaetano (1803), Notizie della Sicilia, n. 1, Notizie piacevoli e curiose o aneddoti dilettevoli, ed eruditi intorno a diversi riti, costumanze, fatti, epoche, di alcune cose rimarchevoli appartenenti a Palermo ed alla Sicilia, Biblioteca Comunale di Palermo, ms. Qq H 43.

Angelini, Jean-Victor (1977), Histoire secrète de la Corse, Paris, Albin Michel.

Aretino, Pietro (1969), Sei giornate. Ragionamento della Nanna e della Antonia, a cura di Giovanni Aquilecchia Bari, Laterza (éd. originale 1534).

Arlacchi, Pino (1992), Gli uomini del disonore. La mafia siciliana nella vita del grande pentito Antonino Calderone, Milano, Mondadori.

Arlacchi, Pino (1994), Addio Cosa Nostra. La vita di Tommaso Buscetta, Milano, Rizzoli.

Atti parlamentari (1993), Camera dei deputati e Senato della Repubblica, XI Legislatura, Commissione d'inchiesta sul fenomeno della mafia e sulle associazioni criminali similari, Parte seconda, Roma, Stabilimenti tipografici Carlo Colomba.

Beccaria, Gian Luigi (1999), Sicuterat. Il latino di chi non lo sa. Bibbia e liturgia nell'italiano e nei dialetti, Milano, Garzanti.

Belando, Vincenzo (1609), Gli amorosi inganni comedia piacevole, David Gilio, Paris.

Bellia Zappalà, Grazia Maria (1981), Un « Mistero di Palermo »: I Beati Paoli di Luigi Natoli, Catania, Facoltà di Lettere e Filosofia-Università di Catania.

Basile, Giambattista (1976), Le Muse napolitane in Lo cunto de li cunti. Le Muse napolitane e le Lettere, a cura di Mario Petrini, Roma-Bari, Laterza (éd. originale 1635).

Benigno, Francesco (2015), La mala setta. Alle origini di mafia e camorra 1859-1878, Torino, Einaudi. Biagi, Enzo (1986), II boss è solo. Buscetta: la vera storia di un vero padrino, Milano, Mondadori.

Bolzoni, Attillo - D’Avanzo, Giuseppe (1993), Il capo dei capi. Vita e carriera criminale di Totò Riina, Milano, Mondadori.

Bridgwater, Patrick (2013), The German Gothic Novel in Anglo-German Perspective, Amsterdam-New York, Rodopi.

Brydone, Patrick (1775), Voyage en Sicile et a Malthe, traduit de l'anglois par M. Demeunier, Amsterdam et Paris, Pissot et Panckoucke, Vol. II.

Burigny, Jean Levesque de, (1790), Storia generale di Sicilia, traduzione illustrata con note, addizioni, tavole cronologiche e continuata sino a' nostri giorni, a cura di Mariano Scasso e Borrello, Palermo, Solli, tomo III.

Carbone, Salvatore - Grispo, Renato, éd. (1969), L'inchiesta sulle condizioni sociali ed economiche della Sicilia (1875-1876), Bologna, Cappelli, vol. II.

Caruso, Gio Battista (1737), Memorie istoriche di quanto è accaduto in Sicilia dal tempo de' suoi primieri abitatori, sino alla coronazione del re Vittorio Amadeo, Palermo, Antonino Gramignani, Parte seconda, vol. I. 
Casadei, Federica (1996), Metafore ed espressioni idiomatiche. Uno studio semantico sull'italiano, Roma, Bulzoni.

Castelli, Raffaele (1882), « Di un mito moderno », Archivio per lo studio delle tradizioni popolari, vol. I.

Castiglione, Francesco Paolo (1987) Indagine sui Beati Paoli, Palermo, Sellerio.

Castiglione, Francesco Paolo (1999), Il segreto cinquecentesco dei Beati Paoli, Palermo, Sellerio.

Ciuni, Roberto (1978), Un secolo di mafia, in R. Romeo (a cura di), Storia della Sicilia, Napoli, Società Editrice, vol. IX.

Coltro, Dino (1994), Santi e contadini. Lunario della tradizione orale veneta, Verona, Cierre.

Cometa, Michele (1999), Il romanzo dell'architettura. La Sicilia e il Grand Tour nell'età di Goethe, RomaBari, Laterza.

Cutrera, Antonino (1917), Cronologia dei giustiziati di Palermo (1517-1819), Palermo, Boccone del povero.

D’Avila, Gilormu (s.d.), Canzoni amorosi et di deversi autori, Biblioteca Comunale di Palermo, ms. 2

QqC 5.

Della Montagna, Giuseppe (alias Gio. Battista Basili palermitanu) (1640), La cuccagna conquistata, poema heroicu in terza rima siciliana, Palermo, Alfonso Dell'Isola.

Di Maria, Vincenzo (1978), I poeti burleschi dal 1500 al 1650, Catania, Tringale.

Dino, Alessandra (2008), La mafia devota. Chiesa, religione, Cosa Nostra, Roma-Bari, Laterza.

Dobrovol'skij, Dmitrij - Piirainen, Elisabeth (2010), « Idioms. Motivation and etymology », Yearbook of Phraseology, vol. 1.

Dufourny, Léon (1991) Diario di un giacobino a Palermo: 1789-1793, a cura di Geneviéve Bautier-Bresc, Palermo, Fondazione Lauro Chiazzese della Sicilcassa [Journal de Léon Dufourny à Palerme, 8 juillet 1789-29 septembre 1793, BNF, ms. Ub 236].

Eco, Umberto (1971), «I Beati Paoli e l'ideologia del romanzo "popolare" », in Natoli Luigi, I Beati Paoli, Palermo, Flaccovio, pp. V-XIII.

Galeano, Giuseppe (alias Pier Giuseppe Sanclemente) (1651), Le Muse siciliane overo Scelta di tutte le canzoni della Sicilia, Palermo, Bisagni, vol. III.

Galluzzo, Lucio - La Licata, Francesco - Lodato, Saverio (1986), Rapporto sulla mafia degli anni '80. Gli atti dell'ufficio istruzione del tribunale di Palermo. Falcone inedito: intervista-racconto, Palermo,

Flaccovio.

Gambetta, Diego (1992), La mafia siciliana. Un'industria della protezione privata, Torino, Einaudi.

Ganci, Massimo (1978), La nazione siciliana, Napoli, Storia di Napoli e della Sicilia Società editrice.

Gomes, Gilormu (1618), Lu bravazzu in lingua siciliana, Palermo, Battista Maringo.

Grasso, Pietro (2012), Liberi tutti. Lettera a un ragazzo che non vuole morire di mafia, Milano, Sperling \& Kupfer.

Hobsbawm, Eric (1995) «Inventer des traditions », Enquête, n. 2.

La Duca, Rosario (1971), Storia e leggenda de «I Beati Paoli » in Natoli Luigi, I Beati Paoli, Palermo, Flaccovio, pp. XXV-XLIII.

Lakoff, George - Johnson, Mark (1980), Metaphors We Live By, Chicago, University of Chicago Press. 
La Platière, Jean-Marie Roland de (1780), Lettres écrites de Suisse, d'Italie, de Sicile et de Malte, Amsterdam, vol. II.

La Porte, Joseph de (1782), Le voyageur françois, ou La connaissance de l'ancien et du nouveau monde, Paris, Cellot, tome XXVIII.

La storia di li fantasimi sunnati d'un viddanu astutu chi durmia cu lu purci ntra l'auricchia (s.d.), Palermo, Emmanuele Ferrer libraro.

Linares, Vincenzo (1836), «I Beati Paoli », Il Vapore, giornale istruttivo e dilettevole, Palermo, Lao e Roberti, Anno III, vol. III, n. 36, 30 décembre 1836.

Linares, Vincenzo (1840), Racconti Popolari, Palermo, Bernardo Virzì.

Lippi Lorenzo (alias Pierleone Zipoli) (1688), Malmantile racquistato, con note di Puccio Lamoni (alias Paolo Minucci), Firenze, S.A.A. alla Condotta.

Marchetti, Pascal (2003), Le Corse dans tous les sens. Chroniques d'identité, Ajaccio, Piazzolla.

M[ayer] chevalier de, (1796) Voyage de Sicile et de quelques parties de la Calabre en 1791, Vienne.

Migliorini, Bruno (1968), « Postilla » a Franca Ageno, « Essere della casa di San Paolo », Lingua Nostra, vol. XXIX.

Minaci, Brasi (1744), Lu Mischinu in signuria ntra la campagna overo Li danni di la villegiatura in pirsuna di lu populu minutu. Cantu in terza rima, Palermu, (sans éditeur).

Moscovici, Serge (1984), The phenomenon of social representations, Farr, Robert - Moscovici, Serge (eds.), Social representations, Cambridge, Cambridge University Press.

Mongitore, Antonino (1871) Diario palermitano delle cose più memorabili accadute nella città di Palermo dal 13 gennaio 1705 al 27 dicembre 1719 in Biblioteca Storica e Letteraria di Sicilia, a cura di Gioacchino Di Marzo, Palermo, Pedone Lauriel, vol. VIII.

Montemagno, Gabriello (2017), L'uomo che inventò $i$ Beati Paoli, Palermo, Sellerio.

Münter, Friedrich (1790), Nachrichten von Neapel und Sicilien: auf einer Reise in den Jahren in den Jahren 1785 und 1786, Copenhagen, Christian Gottlob Proft.

Natoli, Luigi (1971), I Beati Paoli. Grande romanzo storico siciliano, Palermo, Flaccovio.

Nicolaï, Jean-Baptiste (1988), Les sociétés secrètes en Corse, Ajaccio, Cyrnos et Méditerranée.

Palermo, Gaspare (1816), Guida istruttiva per potersi conoscere con facilità tanto dal siciliano che dal forestiere tutte le magnificenze e gli oggetti degni di osservazione della città di Palermo, Palermo, Reale Stamperia, vol. IV.

Pasqualino, Michele (1785) Vocabolario siciliano etimologico, italiano e latino, Palermo, Reale Stamperia, vol. I.

Pitrè, Giuseppe (1875), Fiabe, racconti e novelle popolari siciliani, Palermo, Pedone Lauriel, vol. IV. Pollaci, Nuccio Fedele (1877), « Varietà Palermitane », Nuove effemeridi Siciliane, vol. VI.

Renda, Francesco (1997), Storia della mafia. Come dove, quando, Palermo, Sigma.

Renda, Francesco (1999), I Beati Paoli. Storia, letteratura e leggenda, Palermo, Sellerio.

Riedesel, Johann Hermann von (1771), Reise durch Sizilien und Grossgriechenland, Zürich, Orell, Gessner, Füsslin und Comp. 
Riedesel, Johann Hermann von (1773), Voyage en Sicile et dans la Grande Grèce, adressé par l'auteur a son ami Mr. Winckelmann traduit de l'allemand accompagné de notes du traducteur et d'autres additions intéressantes, Lausanne, Franc. Grasset et Comp.

Russo, Francesco (1987), I Fraticelli in Sicilia nella prima metà del sec. XIV, in Francescanesimo e cultura in Sicilia (secc. XIII-XVI), Palermo, Officina Studi Medievali.

Salomone Marino, Salvatore (1873), Piccolo Dizionario in La Baronessa di Carini. Leggenda storica popolare del sec. XVI in poesia siciliana, Palermo, Pedone Lauriel.

Salomone Marino, Salvatore (1876), « Tradizione e storia », Nuove Effemeridi Siciliane, III serie, vol. IV.

Salomone Marino, Salvatore (1887), Appunti e studi di Storia e Letteratura siciliana tratti da Manoscritti e libri, ms. Archivio Etnostorico Nazionale, Palermo, in Francesco Paolo Castiglione, Il segreto cinquecentesco dei Beati Paoli, Palermo, Sellerio.

Sanuto, Marino (1880) I Diarii, a cura di Rinaldo Fulin, Venezia, Visentini, tomo III.

Sartor Ceciliot, Mario (1995), « Latinismi liturgici nei dialetti », Studi Mediolatini e Volgari, vol. XLI. Schneider, Jan - Schneider, Peter (1994), « Mafia, antimafia, and the question of Sicilian culture », Politics \& Society, vol. 22, n. 2.

Schifano, Jean-Noël (1990), « Un Cyrano sicilien », Le Monde, 22 juin 1990.

Spatafora, Placido (s.d.), Biblioteca Comunale di Palermo, ms. 2 Qq E 31, Dizionario siciliano, vol. III, Ia-Qui.

Stolberg, Friedrich Leopold Graf zu (1794), Reise in Deutschland, der Schweiz, Italien und Sicilien, Königsberg-Leipzig, Nicovlovius, vol. III.

Testa, Francesco (1769), De vita, et rebus gestis Guilelmi II, Monregali, Bentivenga.

Todaro, Pietro (1998), La grotta dei Beati Paoli, in Di Benedetto, Giuseppe - Staacke, Ursula Faconti, Daniela, (a cura di), Interventi di recupero nel centro storico di Palermo, Palermo, Assessorato al centro storico.

Traugott, Elizabeth Closs - Dasher, Richard (2002), Regularity in Semantic Change, Cambridge, Cambridge University Press.

Trasselli, Carmelo (1982), Da Ferdinando il Cattolico a Carlo V, Soveria Mannelli, Rubbettino.

Tribunale di Palermo (1985), Ordinanza - sentenza emessa nel procedimento penale contro Abbate Giovanni + 706, vol. 5 .

Tribunale di Palermo (1993), Corte di Assise, Processo contro Michele Greco + altri.

Tuzet, Hélèn (1955), La Sicile au XVIII siecle vue par les voyageurs étrangers, Strasbourg, Heìtz.

Ullman, Stephen (1962), Semantics. An Introduction to the Science of Meaning, Oxford, Basil Blackwell.

Varchi, Benedetto (1995), L'Hercolano, a cura di Antonio Sorella, Pescara, Libreria dell'Università Editrice, vol. II (éd. originale 1570).

Villabianca, Emanuele Gaetani, Francesco Maria marchese di (1790), Opuscoli Palermitani, tomo IV, Biblioteca Comunale di Palermo, ms., Qq E 90.

Violi, Patrizia (1997), Esperienza e significato, Milano, Bompiani.

Volpes, Nicola (1972), Tenente Petrosino. Missione segreta in Sicilia, Palermo, Flaccovio. 
Wittgenstein, Ludwig, (2004), Recherches philosophiques, tr. fr. Françoise Dastur, Maurice Elie, JeanLuc Gautero, Dominique Janicaud, Élisabeth Rigal, Paris, Gallimard (éd. originale 1953).

\section{NOTES}

1. Pitrè 1875, p. 58. Sauf précision contraire, les citations en langue étrangère sont livrées en version française par la traductrice de cet article.

2. Sur Natoli et son œuvre, cf. Montemagno 2017.

3. Cf. Renda 1972, pp. 377-419.

4. Ganci 1978, p. 180

5. Eco, 1971, p. XXII. Pour une analyse approfondie du roman, cf. Zappalà 1981.

6. Ciuni 1978, p. 379.

7. Renda 1997, p. 188.

8. Natoli 1971, p. 100.

9. Renda 1997, p. 188.

10. La Duca 1971, p. XXVIII.

11. Le Bâtard de Palerme, Paris, Métailié,1990. Schifano 1990, p. 38.

12. Gambetta 1992, p. 184

13. Schneider - Schneider 1994, p. 241.

14. Bolzoni - D'Avanzo 1993 p. 69.

15. Atti parlamentari 1993, p. 1222.

16. Schneider - Schneider, 1994, p. 242.

17. Grasso 2012, p. 32.

18. Galluzzo - La Licata - Lodato 1986, p. 86.

19. Dino 2008, p. 63.

20. Arlacchi 1994, p. 11.

21. Biagi 1986, p. 200.

22. Arlacchi 1992, p. 56.

23. Tribunale di Palermo 1985, p. 717.

24. Idem, pp. 12568-12570.

25. Benigno 2015, p. 379.

26. Hobsbawm 1995, p. 183.

27. Renda 1999, p. 36.

28. Castiglione 1987, p. 72.

29. Castiglione 1999, p. 56.

30. Idem, p. 146.

31. Idem, p. 149

32. Idem, p. 190.

33. Idem, p. 191.

34. Trasselli 1982, p. 716, n. 243.

35. Idem, p. 715.

36. Cf. Renda 1999, p. 10.

37. Marchetti 2003, p. 64.

38. Angelini 1977, p. 229.

39. Marchetti 2003, p. 64. Cf. aussi Nicolaï 1988.

40. Angelini 1977, p. 229.

41. Cf. Beccaria 1999.

42. Cf. Migliorini 1968 et Sartor Cciliot 1995. 
43. Casadei 1996, p. 13.

44. Cf. Dobrovol'skij et Piirainen 2005 e 2010.

45. Cf. Coltro1994, pp. 68-69.

46. Varchi 1995, p. 442.

47. Lippi 1688, p. 25.

48. Idem, p. 459

49. D’Avila s.d., ff. 322.

50. Gomes 1618, p. 12.

51. Belando 1609, p. 23-24.

52. Galeano 1651, p. 143. Les poèmes de Scimeca sont réunis par Di Maria 1978, pp. 170-213.

53. Della Montagna 1640, p. 19.

54. Spatafora s.d., ff. 290v.

55. La storia di li fantasimi sunnati, s.d., p. 12.

56. Minaci 1744, p. 5.

57. Cf. Violi 1997, p. 150.

58. Cf. Ullman 1962 et Traugott - Dasher 2002.

59. Cf. Tuzet 1955.

60. Cf. Cometa 1999.

61. von Riedesel 1773, p. 21-22.

62. Brydone 1775, p. 54.

63. Mongitore 1871, p. 32.

64. Castelli 1882, pp. 261-262.

65. von Riedesel 1771, p. 21, souligné par nous.

66. Cf. Moscovici 1984.

67. La Platière 1780, p. 353.

68. Idem, p. 399.

69. Brydone 1775 , p. 385-386, note A.

70. La Porte 1782, pp. 173-174.

71. Münter 1790, p. 229.

72. Idem, p. 230.

73. Cf. Bridgwater 2013.

74. Stolberg 1794, p. 349.

75. M[ayer] 1796, p. 138.

76. Ibidem

77. Cf. Caruso 1723, pp. 57-83 et pp. 503-523.

78. Caruso 1737, p. 203.

79. Ibidem

80. Testa 1769.

81. Idem, p. 294

82. Ibidem

83. Burigny 1790, p. 318.

84. Ibidem, n. 88.

85. Ibidem

86. Renda 1999, p. 50.

87. Wittgenstein 2004, p. 85.

88. Villabianca 1790 , ff. $47 r-49 r$.

89. Idem, ff. $47 v$.

90. Idem, ff. $48 r$.

91. Villabianca 1790 , ff. 48 r.

92. Idem, ff. $47 v$. 
93. Idem, ff. $49 r$.

94. Ibidem.

95. Idem, ff. $48 v$.

96. Dufourny 1991, pp. 263-364.

97. Alessi 1776, ff. 129r, n. 125.

98. Alessi 1803, ff. 10r, n. 68.

99. Ibidem.

100. Cf. Pollaci 1877, p. 245.

101. A propos des «camere dello scirocco ", les puits thermiques où on se réfugiait lors des fortes chaleurs, cf. Todaro 1998.

102. Salomone Marino 1873, p. 277.

103. Palermo 1816, p. 65.

104. Idem, p. 64.

105. Linares 1836, p. 294.

106. Idem, p. 295.

107. Linares 1840, pp. 193-197.

108. Idem, p. 166.

109. Ibidem.

110. Carbone et Grispo 1969, p. 1139.

111. Volpes 1972, p. 140.

\section{ABSTRACTS}

D'après plusieurs collaborateurs de justice, les origines de la mafia sont à rechercher dans la secte des Beati Paoli, dont les mafieux prétendent être les descendants. Selon la tradition, elle avait pour but de venger les populations des torts infligés par les puissants. Cependant, si en Sicile l'expression «Beati Paoli» renvoie effectivement à cette secte, dans d'autres régions italiennes elle est utilisée en référence à l'abondance ou à une grande quantité. À travers l'analyse de documents d'archives, à partir de textes remontant au XVIe siècle, cet article s'attache à rétablir le sens originaire de cette expression et à vérifier s'il existe un lien entre l'expression et le mythe palermitain des justiciers. Par ailleurs, en retraçant la genèse du mythe, il permet de vérifier si le noyau de la légende populaire provient d'une tradition orale séculaire ou s'il est le résultat du remaniement de divers éléments qui, sédimentés au fil du temps, ont contribué à le structurer.

Secondo vari collaboratori di giustizia le origini della mafia vanno ricercate nella setta dei Beati Paoli, di cui i mafiosi si dichiarano discendenti. Secondo la tradizione, la setta aveva lo scopo di vendicare il popolo per i torti subiti dai potenti. Tuttavia, se in Sicilia l'espressione Beati Paoli si fa riferimento alla setta, in altre regioni italiane è un modo di dire che indica abbondanza o grande quantità. Attraverso analisi d'archivio, a partire da testi cinque-settecenteschi, questo articolo desidera stabilire il più antico significato dell'espressione e verificare se vi sia un legame fra l'espressione e il mito palermitano dei giustizieri. Inoltre, nel tracciare la genesi del mito cerca di appurare se il nucleo della leggenda popolare derivi da da una plurisecolare tradizione orale o se sia il frutto del rimaneggiamento di vari elementi che, sedimentati nel tempo, lo hanno strutturato. 
INDEX

Keywords: Beati Paoli, sect, Vendicosi, avengers, confraterniy, mafia, myth Mots-clés: Beati Paoli, secte, Vendicosi, justiciers, confrérie, mafia, mythe

\section{AUTHORS}

\section{FRANCESCO VALERIO TUMBARELLO}

Francesco Valerio Tumbarello a obtenu une laurea en psychologie clinique à l'Université de Padoue, avec une thèse intitulée Alle origini della mafia. I Beati Paoli fra psicolinguistica e rappresentazioni sociali. Ses recherchent actuelles sont consacrées au crime organisé, notamment aux sociétés criminelles siciliennes au XIXe siècle. 